\title{
Amphetamine and memory for event duration in rats and pigeons: Disruption of attention to temporal samples rather than changes in the speed of the internal clock
}

\author{
ANGELO SANTI, LORRAINE WEISE, and DWAYNE KUIPER \\ Wilfrid Laurier University, Watertoo, Ontario, Canada
}

\begin{abstract}
In Experiment 1, pigeons were trained to match short (2-sec) and long (8-sec) sample durations to red and green comparison stimuli and red and green samples to vertical and horizontal line comparisons. They received injections of $d$-amphetamine $(2.0 \mathrm{mg} / \mathrm{kg})$ or saline, and the delay interval was manipulated $(0,1,3$, and $9 \mathrm{sec})$. In Experiment 2, rats were trained to discriminate short (2-sec) or long (12-sec) durations of either houselight illumination (light group) or tone (tone group), using a choice procedure. During the test phase of each trial, the left and right levers were presented with the cuelight above one of them on (cued lever) while the other was off (uncued lever). For some of the rats, the correct response following the short sample was to press the cued lever, whereas following the long sample, it was to press the uncued lever. This was reversed for the remaining rats. The rats received injections of methamphetamine $(1.5 \mathrm{mg} / \mathrm{kg})$ or saline, and the delay interval was manipulated $(0,1,3$, and $9 \mathrm{sec})$. Memory for color samples in pigeons was not affected by amphetamine. However, for both rats and pigeons, amphetamine reduced the accuracy of event duration memory even at the 0 -sec delay but did not increase the slope of the retention functions. In addition, in neither experiment was there any evidence that amphetamine produced an overestimation of perceived sample duration. Amphetamine predominantly affected temporal memory in both pigeons and rats by disrupting attention to temporal samples rather than by simply increasing the speed of the internal clock.
\end{abstract}

The effects of amphetamine on memory and learning have been assessed in a variety of species (humans, monkeys, rats, mice, pigeons) with a variety of tasks (passive and active avoidance, radial arm maze, delayed matchingto-sample, and various discrimination learning tasks). Amphetamine administered posttraining enhances the storage of information in reference memory (Haycock, van Buskirk, \& Gold, 1977; Janak \& Martinez, 1992; Martinez et al., 1980). However, amphetamine also impairs working memory performance in delayed matching-to-sample and delayed matching-to-position tasks (Bauer \& Fuster, 1978; Dunnett, 1985; Glick \& Jarvik, 1969; Kesner, Bierley, \& Pebbles, 1981). In addition, amphetamine disrupts accuracy on the radial maze task if delays are imposed between successive choices (Beatty, Bierley, \& Boyd, 1984; Beatty \& Rush, 1983; Burešová \& Bureš, 1982; Kesner et al., 1981), but not if there is no delay between successive choices (Burešová \& Bureš, 1982; Eckerman, Gordon, Edwards, MacPhail, \& Gage, 1980). Amphetamine-induced impairment of delayed win-shift

This research was supported by Grant OGPOOD6378 from the Natural Sciences and Engineering Research Council of Canada. The authors thank Linda Parker for her help and David Hemsworth for his technical assistance. Reprints may be obtained from A. Santi, Department of Psychology, Wilfrid Laurier University, Waterloo, ON, Canada N2L 3C5 (e-mail: asanti@machl.wlu.ca). performance on the radial maze is critically dependent on administration of the drug shortly (10-30 min) before the postdelay retention test (Beatty \& Rush, 1983; Burešová \& Bureš, 1982). Posttraining injection of amphetamine given several hours prior to the postdelay retention tests either has no effect if the rats are highly overtrained (Beatty \& Rush, 1983) or improves performance if the rats are less highly trained (Packard \& White, 1989; Strupp, Bunsey, Levitsky, \& Kesler, 1991).

Interpretation of impairments produced by amphetamine is complicated by the well-known effects of the drug on motor responses and appetite (Beninger, 1983; Fischman, 1987; Rebec \& Bashore, 1984). For example, Beatty and Rush (1983) have argued that amphetamineproduced deficits in radial maze performance are best explained by the changes in motor activity and appetite produced by the drug. However, some disruptive effects of amphetamine cannot be explained in this way. Bushnell and Levin (1993) have directly compared the effects of amphetamine on working memory (accuracy of spatial delayed nonmatching-to-position), reference memory (accuracy of visual discrimination), and motor function in rats. Amphetamine reduced the accuracy of nonmatchingto-position without significantly affecting visual discrimination accuracy or motor function. This suggests that there is an enhanced sensitivity of working memory tasks to disruption by amphetamine, a finding which parallels the re- 
sults already well-established for eholinergic blockade (Beatty \& Bierley, 1985; Burešová, Bolhuis, \& Bureš, 1986; Santi, Bogles, \& Petelka, 1988; Wirsching, Beninger, Jhamandas, Boegman, \& El-Defrawy, 1984). In addition, as in the research literature on anticholinergics, Bushnell and Levin (1993) found that the disruptive effects of amphetamine were delay independent. They concluded that amphetamine did not disrupt the maintenance of sample information as much as its encoding prior to the delay.

As indicated above, the effects of amphetamine on various tasks, such as delayed matching-to-position and radial arm maze, are complexly determined by several factors. This also appears to be true with respect to amphetamine's effect on animals' ability to perceive and remember time. Church (1984) has proposed a model known as the internal clock to depict how an animal perceives and remembers the duration of an event and decides whether it is comparable to other, reinforced, durations. Church's model comprises two stages: the clock stage, consisting of the pacemaker, switch, and accumulator; and the memory stage, which includes both reference and working memory. Closure of the switch allows pulses, dispatched by the pacemaker, to travel to an accumulator. The accumulated pulses are stored on a trial-by-trial basis in working memory. The count in working memory is compared with previously reinforced durations stored in reference memory, and a response decision is made. Various experimental manipulations, such as a drug, may affect one, but not the other, of the two stages, or may affect both.

Researchers have used the point of subjective equality (PSE), or that point at which an animal chooses the response alternative reinforced following the long duration $50 \%$ of the time, to study animals' perception of event duration. Maricq, Roberts, and Church (1981) trained rats to choose between either the left or the right lever following blackouts of different durations. Although a total of seven signals of different durations were used, the two extreme signal durations were the only ones that were rewarded following a correct choice. Three groups of rats were each exposed to a different range of signal durations. On saline test sessions, the PSE was close to the geometric mean between the two extreme signal durations. Injections of $1.5 \mathrm{mg} / \mathrm{kg}$ of methamphetamine disrupted the accuracy of temporal discriminations generally. In addition, it was found that methamphetamine shifted the PSE to the left by approximately $10 \%$ relative to the PSE on saline control sessions. Maricq et al. (1981) reasoned that this was demonstrative of a change in clock speed, because the change in the PSE was positively related to the signal range being timed. In fact, they suggested that methamphetamine increased the clock speed by approximately $10 \%$. Maricq and Church (1983) replicated the finding of a leftward shift in the PSE by methamphetamine. In addition, they demonstrated that haloperidol, a dopamine-receptor blocker, shifted the PSE rightward, whereas a combination of methamphetamine and haloperidol produced the same PSE as that found on saline control sessions.
Using similar procedures, Meck (1983) provided further support for the hypothesis that amphetamine affects the clock stage of the internal clock. He reasoned that if the clock stage was affected by a drug, the effects of that drug would be attenuated with repeated exposure since the rats would learn to rescale the durations. In addition, abrupt termination of exposure to the drug would result in a shift of the psychophysical function in a direction opposite to that originally produced by the drug. However, if memory was affected, the shift in the psychophysical function would be permanent, and termination of chronic exposure would not result in a rebound effect. Meck found that $1.5 \mathrm{mg} / \mathrm{kg}$ of methamphetamine shifted the PSE to the left by about $10 \%$. When methamphetamine administration was terminated, the PSE was shifted to the right. Haloperidol produced effects complementary to those of methamphetamine but in the opposite direction. Meck concluded that the effective level of dopamine determines the setting of clock speed in the rat, with methamphetamine increasing clock speed and haloperidol decreasing it.

In these studies of methamphetamine and time estimation in rats (Maricq \& Church, 1983; Maricq et al., 1981; Meck, 1983), the psychophysical functions were calculated on the basis of responses faster than the mean latency of responses, with a correction for responses not controlled by time. This method of data analysis appears to be critical to the demonstration of a "choose-long" response bias under amphetamine, and it allows Meck (1983) to argue that methamphetamine and haloperidol selectively adjust the speed of the clock without affecting memory processes. Other studies of amphetamine's effects on temporal discriminations in both rats (Rapp \& Robbins, 1976) and pigeons (Stubbs \& Thomas, 1974) have included the responses of all trials in their analyses. Somewhat surprisingly in these studies, amphetamine does not produce a choose-long response bias. Rapp and Robbins trained rats to make a left lever response following a 3-sec tone and a right response following a 7-sec tone. At the highest dose $(0.8 \mathrm{mg} / \mathrm{kg})$ of $d$-amphetamine administered, accuracy was disrupted and 4 out of 5 rats exhibited a bias toward "short" responses. Stubbs and Thomas trained pigeons to peck a red comparison stimulus following short-duration samples and a green comparison stimulus following long-duration samples. They found that amphetamine had greater effects on longer sample durations, regardless of the absolute lengths of those durations. That is, amphetamine produced a choose-short response bias like that produced by increasing the length of delay intervals (Spetch, 1987).

These results indicate that amphetamine-induced impairment of temporal discrimination performance involves more than simply direct influences on internal clock processes. Additional evidence for this comes from Spetch and Treit (1984). Contrary to both Stubbs and Thomas (1974) and Rapp and Robbins (1976), they found that, in pigeons, amphetamine produced a choose-long bias at a 0 -sec delay, suggesting a lengthening of perceived time. However, they also found that under amphetamine at 
longer delays there was no difference in accuracy between short and long samples. Accuracy was near chance levels. Spetch and Treit suggested that, in addition to accelerating the rate of the internal clock, amphetamine caused a general decrement in short-term memory. Meck (1983) also noted that these drugs may not exclusively affect processes related to time discrimination. The memory disruption hypothesis receives support from previous research on nontemporal memory tasks which demonstrates that working and reference memory can be affected by amphetamine (Bauer \& Fuster, 1978; Beatty et al., 1984; Beatty \& Rush, 1983; Burešová \& Bureš, 1982; Dunnett, 1985; Glick \& Jarvik, 1969; Haycock et al., 1977; Janak \& Martinez, 1992; Kesner et al., 1981; Martinez et al., 1980). However, in pigeons, a disruptive effect of amphetamine on nontemporal memory has not been well documented. For example, Teal and Evans (1982) found that doses of amphetamine between 0.03 and $1.0 \mathrm{mg} / \mathrm{kg}$ had no effect on the accuracy of color delayed matchingto-sample in pigeons. A dose of $1.0 \mathrm{mg} / \mathrm{kg}$ is sufficient to disrupt the accuracy of temporal discriminations in both pigeons (Stubbs \& Thomas, 1974; Spetch \& Treit, 1984) and rats (Rapp \& Robbins, 1976).

The present studies were designed to extend the current knowledge of the effects of amphetamine on both time perception and memory for durations in both pigeons (Experiment 1) and rats (Experiment 2). In both experiments, the effect of amphetamine was assessed at delays of $0,1,3$, and $9 \mathrm{sec}$. This permits an assessment of the effect of amphetamine on time perception at the 0 -sec delay, as well as of its effects on memory for durations at longer delays. Only Spetch and Treit (1984) have examined the effects of amphetamine on memory for durations.

\section{EXPERIMENT 1}

Pigeons were trained to match short (2-sec) and long (8-sec) sample durations to red and green comparison stimuli and red and green samples to vertical and horizontal line comparisons. Subsequently, they received injections of $d$-amphetamine $(2.0 \mathrm{mg} / \mathrm{kg})$ or saline, and the delay interval (DI) was manipulated $(0,1,3$, and $9 \mathrm{sec})$. This permitted an examination of drug effects on both temporal and nontemporal memory in pigeons. Previous timing research in rats suggested a direct effect of amphetamine on clock processes (e.g., Maricq \& Church, 1983; Maricq et al., 1981; Meck, 1983), whereas other research indicated that amphetamine has additional effects both on temporal discriminations (Rapp \& Robbins, 1976; Spetch \& Treit, 1984; Stubbs \& Thomas, 1974) and on other nontemporal memory tasks (e.g., Beatty \& Rush, 1983; Bushnell \& Levin, 1993). If amphetamine simply increased the speed of the internal clock, it was expected that a choose-long bias would be demonstrated. If, on the other hand, amphetamine affects memory generally as well as clock processes specifically, it was expected that a choose-short response bias would be observed, because this bias is typically produced when memory is challenged by increases in the length of DIs. The present study was also intended to determine whether a larger dose of amphetamine than that used in Teal and Evans (1982) would have an effect on delayed matching of color samples in pigeons. Perhaps temporal discriminations would be more sensitive to disruption by amphetamine than would nontemporal discriminations.

\section{Method}

Subjects. Eight adult male White Carneaux pigeons, maintained at approximately $80 \%$ of their ad-lib weights and housed individually with constant access to grit and water, served as subjects. The colony room was on a 12:12-h light:dark cycle, with fluorescent lights on from 8:00 a.m. to 8:00 p.m. Subjects were tested 5 days a week during the light cycle. All birds had extensive experience with delayed matching tasks, including memory for event duration.

Apparatus. Four Coulbourn modular operant test cages (Model E10-10), housed individually in isolation cubicles (Model 10-20), were used. The cubicles were equipped with a ventilation fan and baffled air intake exhaust systems. Each test cage was equipped with three horizontally aligned clear plastic keys, behind which projectors could display stimuli (red, green, or yellow field; a white vertical, diagonal, or horizontal line on a black background, or a black dot on a white background) onto a frosted rear projection screen (Coulbourn Model E21-18). Directly below the center key was a $5.7 \times 5 \mathrm{~cm}$ opening that provided access to a hopper containing mixed grain (Coulbourn Model E14-10). A houselight was located $6.5 \mathrm{~cm}$ above the center key and was installed in such a way that the light was directed upward to reflect from the top of the cage (Coulbourn Model E11-01 with bulb SL1819X). All experimental events and response measures were arranged and recorded by a microcomputer system located in an adjacent room.

Procedure. All birds had received extensive prior delayed matching training with color (red and green fields) and temporal (2-or 8-sec illumination of crossed diagonal lines) samples. Color sample stimuli were followed by line-tilt comparison stimuli (vertical and horizontal lines), and temporal sample stimuli were followed by color comparison stimuli (red and green fields). Position of the comparison stimuli was counterbalanced over trials. Each trial began with the warning stimulus (black dot on a white background) presented on the center key. A single peck to it resulted in presentation of one of four sample stimuli on the center key. The color samples were presented for $4 \mathrm{sec}$, because this is an approximate estimate of the PSE between 2- and 8-sec temporal samples for pigeons (Spetch $\&$ Wilkie, 1983). Immediately following the offset of the sample stimulus, the comparison stimuli were presented. For all birds, a response to the red comparison was correct following a 2-sec sample, and a peck to the green comparison was correct following an 8-sec sample. For 5 birds, a peck to the vertical line comparison following a red sample and a peck to the horizontal line comparison following a green sample was correct. For the remaining 3 birds, the relationship between the color sample and the correct line comparison stimulus was reversed. A single peck to the comparison stimuli turned them off and if correct permitted 3-sec access to mixed grain. Incorrect responses to the comparison stimuli produced a 3-sec blackout, followed immediately by re-presentation of the same sample stimulus and comparison stimulus configuration. A correct response on a correction trial produced 3-sec access to mixed grain, although only the choice response on the initial (noncorrection) trial was used to calculate matching accuracy. When necessary, supplementary feedings of Purina Pigeon Chow occurred after the experimental sessions.

Within each block of eight trials, all combinations of the four sample stimuli with the various comparison stimuli on the left and right keys occurred once. The order of presentation was randomized individually for each bird. All birds received 72 trials per session. The intertrial interval (ITI) was a constant $20 \mathrm{sec}$ in length. Prior 
to drug testing, the performance of all birds was at $80 \%$ or better on both color and temporal sample trials.

On each session of drug testing, the birds received either an injection of saline or an injection of $d$-amphetamine sulfate at a dose of $2.0 \mathrm{mg} / \mathrm{kg}$. This particular dose was selected because it had been used in prior time discrimination research with pigeons (Spetch \& Treit, 1984; Stubbs \& Thomas, 1974), and because it was below the dosage range that produces gross side effects such as stereotypy (Goodman, 1981). All injections were made into the pectoral muscle $10 \mathrm{~min}$ prior to each test session in a volume of $1.0 \mathrm{ml} / \mathrm{kg}$ of body weight. As in the Spetch and Treit (1984) study, each bird received $d$-amphetamine and saline on alternate days, with 4 birds receiving each on any given test session. A total of 18 test sessions (9 saline, 9 amphetamine) was administered. Each drug test session consisted of 96 trials, within which 18 trials for each sample occurred at the 0 -sec delay and 2 trials for each sample occurred at each of the other delays $(1,3$, and $9 \mathrm{sec})$. This distribution of delays was used so that the reference memory of durations of temporal samples and their associations with the comparison stimuli established during 0 -sec delay training would remain relatively stable during testing (Spetch \& Wilkie, 1983). There was no illumination in the test chamber during the delay intervals. All other parameters were the same as those described previously, but without a correction procedure.

\section{Results and Discussion}

The mean percentage of correct matching performance for both temporal and color samples during drug testing sessions at the various DIs is shown in Figure 1. Pigeons performed more accurately on trials with color samples than on trials with temporal samples $[F(1,7)=$ $8.27, p<.05]$, and accuracy under both drug conditions decreased as the DI increased $[F(3,21)=63.35, p<.01]$. Overall accuracy with amphetamine was significantly lower than accuracy with saline $[F(1,7)=15.29, p<.01]$. However, there was a significant interaction of drug $X$ dimension $[F(1,7)=7.22, p<.05]$. This was due to the fact that amphetamine did not disrupt accuracy on trials with color samples $(F<1)$, but it did significantly reduce accuracy on trials with temporal samples $[F(1,7)=$

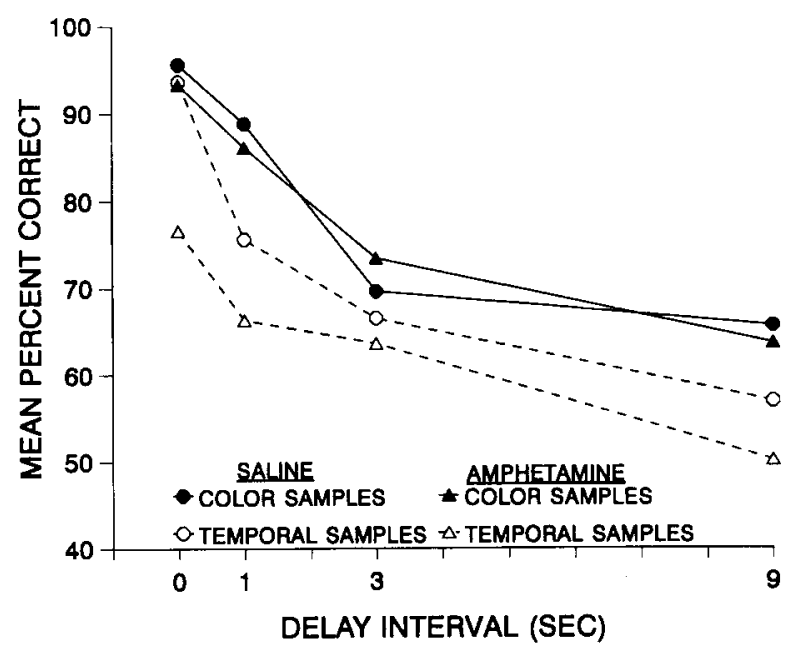

Figure 1. Mean percent of correct matching responses as a function of drug (saline or amphetamine) and sample dimension (temporal or color) at delays of $0,1,3$, and 9 sec.
$14.70, p<.01]$. Moreover, there was no significant difference in accuracy between the color and temporal samples when pigeons were treated with saline $[F(1,7)=3.49$, $p>.10]$. There was, however, a difference in accuracy between the two dimensions when the animals were treated with amphetamine $[F(1,7)=11.06, p<.05]$, such that the animals performed with lower accuracy on trials with temporal samples. The analysis of variance also revealed a second interaction of drug $\times \mathrm{DI}[F(3,21)=6.63, p<$ $.01]$. This interaction was due to the occurrence of a significant drug effect at the $0-, 1-$, and 9-sec DIs, $[F \mathrm{~s}(1,7)=$ $16.78,11.88$, and $13.16, p s<.05]$, but not at the $3-\mathrm{sec}$ DI $(F<1)$.

The data obtained on trials with color samples replicates and extends the finding of Teal and Evans (1982) at a higher dosage of amphetamine. Amphetamine does not disrupt the accuracy of matching with color samples when injected at doses up to $2.0 \mathrm{mg} / \mathrm{kg}$. It does not appear to matter whether the comparison stimuli themselves are colors as in Teal and Evans (1982) or line orientations as in the present study. However, this same dose of amphetamine does disrupt the accuracy of matching with temporal samples. This finding is consistent with the results of previous studies, which indicate that amphetamine reduces the overall accuracy of temporal matching at a 0sec delay in both pigeons and rats (Rapp \& Robbins, 1976; Spetch \& Treit, 1984; Stubbs \& Thomas, 1974).

In order to examine the specific way in which amphetamine reduces the accuracy of temporal memory, additional analyses were conducted with sample duration (short or long) as a factor. These data are displayed in Figure 2. Overall accuracy was significantly lower with amphetamine $(64.0 \%)$ than with saline $(73.2 \%)[F(1,7)=$ $14.70, p<.05]$, and it declined with increases in DI $[F(3,21)=34.63, p<.01]$. In addition, accuracy was significantly higher on short-sample trials than on longsample trials $[F(1,7)=9.63, p<.05]$. None of the interactions involving sample duration were statistically significant. Under both drug conditions, the pigeons show a bias to choose the comparison stimulus paired with the short sample, suggesting that amphetamine does not simply cause the pigeons to overestimate time. These findings agree with the results of Rapp and Robbins (1976) and Stubbs and Thomas (1974), but not with those of Spetch and Treit (1984).

It seems unlikely, in light of these findings, that amphetamine simply increases the speed of the internal clock, since there is no evidence of a choose-long effect or even a decrease in the magnitude of the choose-short effect. Furthermore, the effect does not seem to be simply the result of changes in motor activity, appetite, or attention, since a similar decline in accuracy is not seen with the color samples. It is also clear that amphetamine's disruption of temporal matching is not delay dependent. Despite differences in experimental paradigm and species, the present results lead to a similar conclusion as reached by Bushnell and Levin (1993). Amphetamine does not disrupt the maintenance of information derived from the sample stimulus as much as the encoding of that infor- 


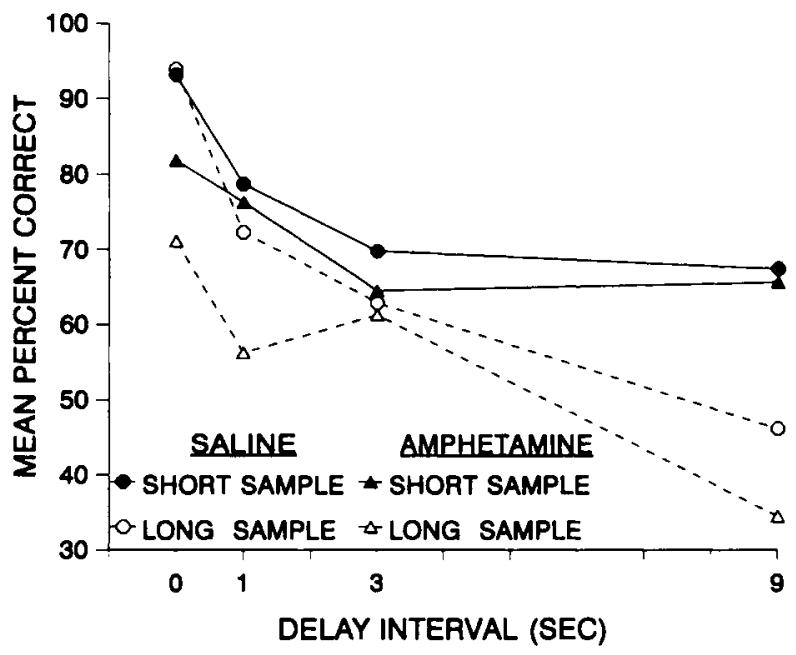

Figure 2. Mean percent of correct matching responses as a function of drug (saline or amphetamine) and sample duration (short or long) at delays of $0,1,3$, and $9 \mathrm{sec}$.

mation prior to the delay. The discrepancy between the performance of amphetamine-treated pigeons on color and temporal samples may be the result of a difference in ease with which these samples are encoded.

\section{EXPERIMENT 2}

The present experiment was designed to further explore the effects of amphetamine on temporal perception and memory for durations. Rats were trained to discriminate short $(2-\mathrm{sec})$ or long $(12-\mathrm{sec})$ durations of either houselight illumination (light group) or tone (tone group), using a choice procedure. The modality of the sample was varied because previous research in our lab has indicated differences in the nature of the systematic memory errors displayed as a function of the modality of the event duration (Santi, Weise, \& Kuiper, 1995).. During sessions in which the DI was varied, rats received injections of methamphetamine $(1.5 \mathrm{mg} / \mathrm{kg})$ or saline. In accordance with Meck's (1983) analysis of the internal clock and pharmacological manipulations, if amphetamine affects the memory stage, the effect of the drug should be permanent, and no rebound would occur upon termination of drug testing. In contrast, if amphetamine affects the clock stage, the effects should be attenuated with repeated exposure to the drug, and when tested in the absence of the drug, subjects should demonstrate a rebound effect, which would be opposite in direction to the initial effect of amphetamine. Furthermore, if amphetamine works by only increasing the speed of the internal clock, then it should produce a choose-long bias during drug testing.

\section{Method}

Subjects. Fifteen male Sprague-Dawley rats, maintained at approximately $80 \%$ of their ad-lib weights and individually housed, served as subjects. Five of these were eliminated because of illness or inability to perform the task. The colony room was on a 12:12-h light:dark cycle, with fluorescent lights on from 8:00 a.m. to 8:00 p.m. Subjects were tested 5 days a week during the light cycle. They were given free access to water at all times. All subjects had previous experience with the current delayed matching-to-sample task in which ITI and DI manipulations had been investigated. Furthermore, all rats had served in a brief drug study that did not involve operant training.

Apparatus. Four Coulbourn operant chambers (Model E 10-10) were individually housed in isolation cubicles (Model E10-20), each of which was equipped with both a ventilation fan and a baffled air intake exhaust system. On the front wall of each chamber were two retractable levers (Model E23-07), approximately $2.5 \mathrm{~cm}$ from the floor of the cage and $14 \mathrm{~cm}$ apart. Directly above each lever was a 7.5-W opaque white jewel lamp. Between the two levers was a 45 mg pellet feeder (Model E14-06), which provided access to dustless precision pellets. A houselight (Coulbourn Model E11-01 with bulb SL1819X) was in place $6.5 \mathrm{~cm}$ above the pellet feeder and was positioned so that its light reflected from the top of the cage. A 2.9-kHz tone module (Model E12-02) was positioned $2 \mathrm{~cm}$ below the houselight. All events and responses were arranged and recorded by a microcomputer system located in the same room.

Procedure. All rats had been previously trained in the delayed matching procedure, in which presentation of either the houselight $(N=5)$ or the tone $(N=5)$ served as the sample stimulus. Both the light and tone group were given one daily session of 72 trials on 5 days per week. The chamber was darkened throughout a session, except for the occasional experimental presentations of houselight, cue lights, and magazine light. Each trial was initiated by onset of the houselight for 2 or $12 \mathrm{sec}$ in the light group or by the onset of the tone for 2 or $12 \mathrm{sec}$ in the tone group. Termination of the sample was followed by a 2 -sec period during which the cuelight above either the left or the right lever was illuminated prior to entry of the levers into the chamber. This will be referred to as the 0-sec delay condition, because onset of the cuelight immediately after sample termination provided the rat with information concerning the correct spatial location to respond to once the levers were inserted. Within each block of four trials, all combinations of the two event durations with the two test conditions (i.e., cued left-uncued right, or uncued left-cued right) occurred once. The order of presentation was randomized individually for each rat. For 2 rats in the light group and 1 in the tone group, responding on the cued lever was correct after the 2-sec signal and responding on the uncued lever was incorrect; following a 12-sec signal, the uncued lever was correct and the cued lever was incorrect. These contingencies between event duration and correct lever were reversed for 3 rats in the houselight group and 4 rats in the tone group. A response to the correct lever turned off the cue light, retracted both levers, and resulted in delivery of a food pellet. Pellet delivery produced an audible "click," and the light in the magazine was illuminated for $0.5 \mathrm{sec}$. A response to the incorrect lever turned off the cue light and retracted both levers. A correction procedure was used throughout training. Following an incorrect response, a 5 -sec delay period occurred, and this was followed immediately by re-presentation of the same sample duration and the same cued/uncued-lever configuration. A correct response on a correction trial turned off the cue light, retracted both levers, and resulted in delivery of a food pellet. Only the choice response on the initial (noncorrection) trial was used to calculate matching accuracy. Following delivery of the reinforcer, an ITI of $25 \mathrm{sec}$ was spent in darkness. Prior to drug testing, all rats were able to maintain accuracy levels of $80 \%$ or greater.

For purposes of drug testing, the 10 rats were divided into groups so that one group received 15 days of testing with saline followed by 15 days with amphetamine ( $N=4: 2$ light and 2 tone) and vice versa for the second group ( $N=6: 3$ light and 3 tone). Twenty minutes prior to each test session, each rat was given an intraperitoneal (i.p.) injection of methamphetamine at a dose of $1.5 \mathrm{mg} / \mathrm{kg}$ or saline in a volume of $1.0 \mathrm{ml} / \mathrm{kg}$ and was immediately placed in the operant chamber. This particular dose and form of amphetamine was selected because it had been used in prior timing research with rats 
(Maricq \& Church, 1983; Maricq et al., 1981; Meck, 1983). Each drug test session consisted of two blocks of 36 trials each. Within each block of 36 trials, there were 12 short and 12 long samples tested at the 0 -sec delay and two short and two long sample durations tested at each delay of 1,3 , and $9 \mathrm{sec}$. This distribution of delays was used so that the reference memory of durations of temporal samples and their associations with the comparison stimuli established during 0 -sec delay training would remain relatively stable during testing (Spetch \& Wilkie, 1983). The DIs represented periods of darkness between sample termination and onset of the cue light for 2 sec prior to lever entry. All other parameters were the same as training sessions, but without a correction procedure.

\section{Results and Discussion}

The data were averaged across the 15 test sessions for saline and the 15 test sessions for amphetamine. They are shown in the upper half of Figure 3 for the light group and in the bottom half for the tone group. An analysis of variance was conducted, with modality (light vs. tone) and order (saline-amphetamine vs. amphetamine-saline) as between-subjects factors and with drug, sample duration, and DI as within-subjects factors. Accuracy with amphetamine was significantly lower than with saline $[F(1,6)=38.91$, $p<.01]$. Accuracy declined as the DI increased $[F(3,18)$
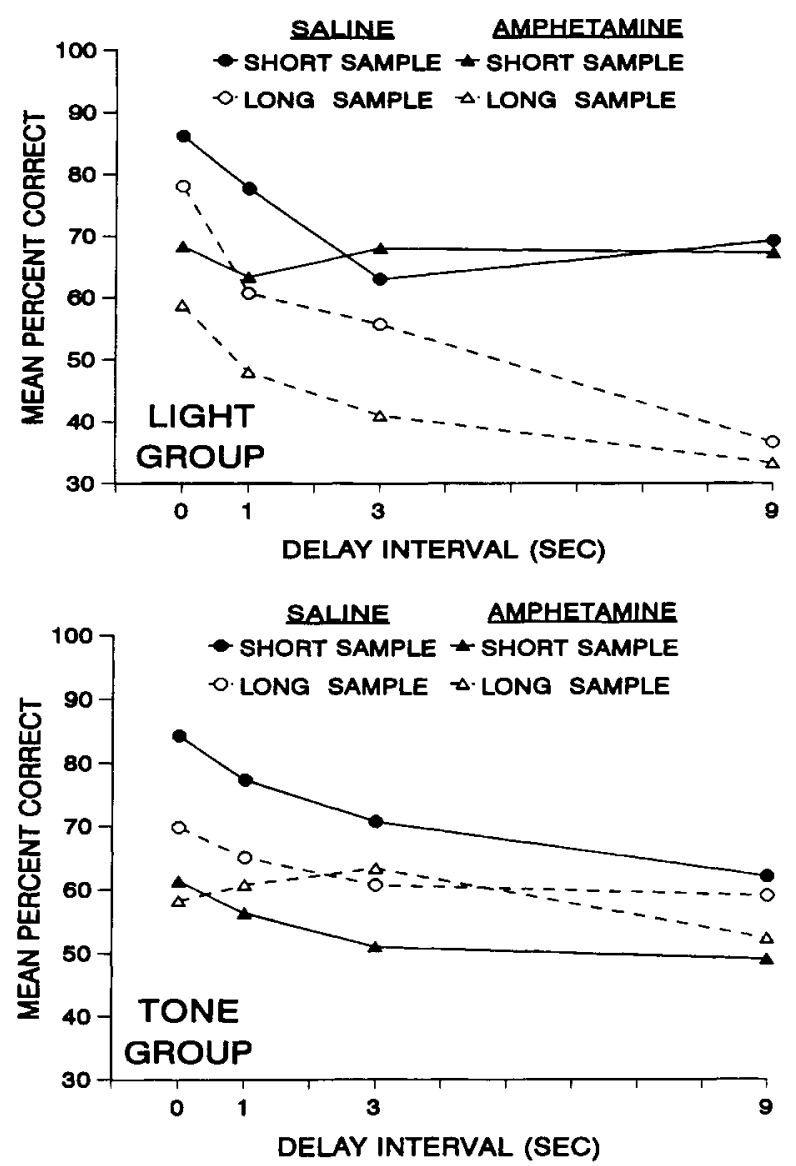

Figure 3. Mean percent of correct matching responses as a function of drug (saline or amphetamine) and sample duration (short or long) at delays of $0,1,3$, and $9 \mathrm{sec}$ for rats trained with durations of light (top) and rats trained with durations of tone (bottom).
$=40.78, p<.01]$, and it was higher on short sample trials than on long sample trials $[F(1,6)=17.10, p<.01]$. The analysis of variance revealed several interactions which involved the modality factor, including modality $\times$ DI $[F(3,18)=3.37, p<.05]$, modality $\times$ sample duration $[F(1,6)=9.25, p<.05]$, modality $\times$ DI by sample duration $[F(3,18)=4.32, p<.05]$, and modality $\times$ drug $\times \mathrm{DI} \times$ sample duration $[F(3,18)=3.08, p=.05]$.

A simple main effects analysis conducted on the highest order significant interaction indicated that for the light group a choose-short effect was present both under saline $[F(3,18)=3.19, p=.05]$ and under amphetamine $[F(3,18)=5.92, p=.01]$. In addition, there was a significant DI $\times$ sample duration interaction $[F(3,18)=$ $4.75, p=.01]$ for the light group. Accuracy on short and long sample trials did not differ significantly at the 0 -sec DI $[F(1,6)=2.12, p=.20]$; however, at the 1-, 3-, and 9-sec DIs, accuracy was significantly greater on short sample trials than on long sample trials $\left[F_{\mathrm{s}}(1,6)=11.98\right.$, 8.62 , and $36.50, p s<.05]$. For the tone group, although there was a trend toward higher accuracy on short than on long sample trials at the short delays under saline, none of the effects involving sample duration were statistically significant. Consequently, for the light group, a statistically significant choose-short effect was observed under both saline and amphetamine, whereas for the tone group no significant choose-short effect was obtained under either saline or amphetamine.

A significant drug $\times$ DI interaction was obtained in both the light group $[F(3,18)=13.16, p<.01]$ and the tone group $[F(3,18)=3.57, p<.05]$. In both groups, amphetamine significantly reduced accuracy. For the light group, accuracy with amphetamine was significantly lower than under saline at the 0 -sec $[F(1,6)=$ $22.48, p<.01]$ and $1-\sec \operatorname{DIs}[F(1,6)=58.03, p<.01]$, but not at the $3-\sec [F(1,6)=3.54, p=.11]$ or $9-\sec (F<1)$ DIs. For the tone group, accuracy under amphetamine was significantly lower than under saline at all DIs $[F \mathbf{s}(1,6)=$ $19.36,51.09,10.94$, and $7.83, p \mathrm{~s}<.05]$.

The only statistically significant effect in the analysis of variance involving the order factor was an order $X$ drug $\times$ DI interaction $[F(3,18)=4.14, p=.01]$. It is shown in Figure 4. During both saline and amphetamine testing sessions, there were no statistically significant differences between the group receiving saline treatment first (saline-amphetamine) and the group receiving amphetamine treatment first (amphetamine-saline). However, the two groups did differ slightly with respect to the DIs at which significant drug effects occurred. For the saline-amphetamine group, amphetamine significantly impaired performance at the $0-\sec [F(1,6)=29.31, p<$ $.01], 1-\sec ,[F(1,6)=51.13, p<.001]$, and 3-sec DIs, $[F(1,6)=12.11, p<.05]$, but not at the 9-sec DI $(F<1)$, where the rats performed poorly under both saline and amphetamine. For the amphetamine-saline group, amphetamine significantly decreased accuracy at the 0 -sec $[F(1,6)=15.40, p<.01], 1-\sec [F(1,6)=58.39, p<$ $.001]$, and 9-sec DIs $[F(1,6)=6.31, p<.05]$, but not at the 3-sec DI $[F(1,6)=3.59, p>.10]$. 


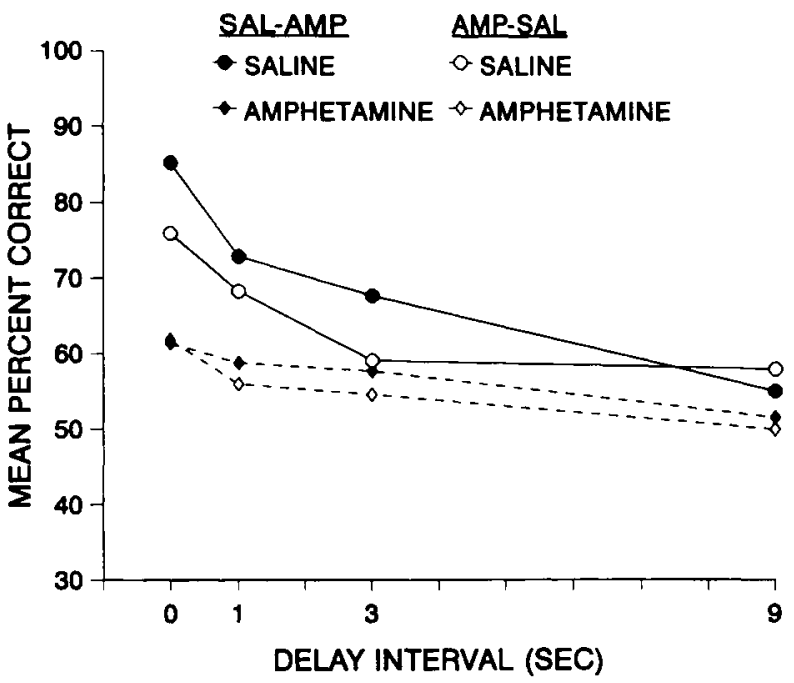

Figure 4. Mean percent of correct matching responses as a function of the between-subjects variable of drug treatment order (salineamphetamine or amphetamine-saline) and the within-subjects variable of drug condition (saline or amphetamine) during testing at delays of $0,1,3$, and $9 \mathrm{sec}$.

In order to determine whether there were any changes in performance across test sessions, the test data were collapsed into blocks of five sessions, and the data for saline and amphetamine test blocks were analyzed separately. On saline test sessions, there was a significant modality $\times$ block $\times$ delay $\times$ sample duration interaction $[F(6,48)=$ 2.30, $p=.05]$. This interaction is shown in Figure 5 . The data in the upper half of the figure indicate that for the light group the choose-short effect was attenuated over test blocks. A significant choose-short effect was obtained in Blocks 1 and $2[F \mathrm{~s}(3,24)=6.61$ and $3.38, p<$ $.05]$, but not in Block $3(F<1)$. The data in the bottom half of the figure show that for the tone group there was little change in accuracy over test blocks. For the tone group, accuracy declined as the DI increased $[F(3,24)=$ $8.54, p<.01]$; however, neither the effect of blocks nor the interaction of blocks with any other factor was significant.

An analysis across test blocks for the amphetamine data revealed a significant modality $\times$ delay $\times$ sample duration interaction $[F(3,24)=5.82, p<.01]$ as a result of the presence of a choose-short effect in the light group, but not in the tone group. However, unlike for the saline data, there was no evidence of any change in performance across test blocks. Neither the main effect of blocks nor any interaction involving blocks was statistically significant for amphetamine test sessions.

The results of the present experiment are inconsistent with the notion that amphetamine simply increases the speed of the internal clock. Neither the light group nor the tone group displayed any evidence of a choose-long bias under amphetamine. The amphetamine data for the light group are consistent with those previously reported for rats (Rapp \& Robbins, 1976) and pigeons (Stubbs \& Thomas, 1974), in that there is a drop in accuracy under amphetamine and the presence of a choose-short effect. The data for the tone group also reveal a significant drop in accuracy under amphetamine, but no systematic response bias was evident in this group either under saline or under amphetamine. In addition, there is no evidence that amphetamine disrupts the maintenance of information derived from the sample stimulus. If it acted in this fashion, one would anticipate greater effects of amphetamine at the longer delay intervals. This did not occur. Instead amphetamine produced marked disruption at the short delays in both the light and the tone group. These findings seem to be consistent with the hypothesis that amphetamine produces a general disruption of the encoding of information prior to the delay.

\section{GENERAL DISCUSSION}

Under control conditions in both studies, pigeons and rats in the light group displayed a choose-short bias following temporal samples when the retention interval was increased. The retention functions displayed by the rats in the tone group failed to reveal any significant response bias. These findings replicate those which we have previously obtained (Santi et al., 1995). The presence or ab-
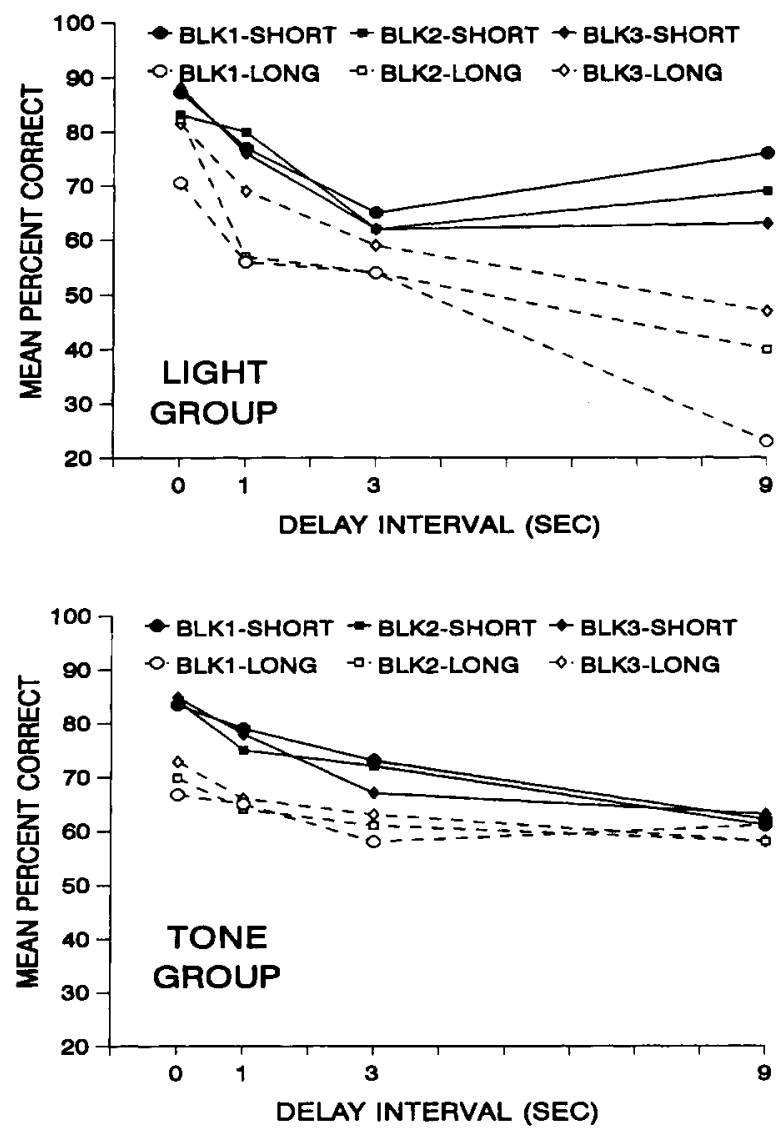

Figure 5. Mean percent of correct matching responses as a function of block $(1,2$, or 3$)$ and sample duration (short or long) during saline test sessions at delays of $0,1,3$, and 9 sec for rats trained with durations of light (top) and rats trained with durations of tone (bottom). 
sence of a "choose-short" bias has been used as a diagnostic indicator of the nature of the coding strategy used. Its presence has been used to argue that there is an isomorphism between the temporal properties of the sample and the working memory code for that sample, such that the remembered durations in working memory undergo subjective shortening during the DI (Spetch \& Wilkie, 1983). This retrospective and analogical coding of temporal samples is presumably responsible for the "chooseshort" effect (Grant \& Spetch, 1991, 1993; Spetch \& Sinha, 1989; Wilkie \& Willson, 1990). Consequently, the data from the control conditions of the present study suggest that rats retrospectively code visual samples of different durations in terms of perceived duration, but not auditory samples of different durations. Pigeons also appear to retrospectively code visual samples of different duration, but no data are available on their memory for auditory event durations.

Regardless of the way in which pigeons and rats in the light or the tone group coded temporal samples, the effect of amphetamine was similar in all groups. Amphetamine reduced the overall accuracy of responding even at a 0 -sec delay. In none of the groups was there any evidence of the choose-long bias that would be expected if amphetamine simply increased the speed of the internal clock. If a choose-short bias was evident under saline conditions, as it was for pigeons and the rats in the light group, then a choose-short bias was evident under amphetamine conditions, only the overall accuracy was significantly lower. If a choose-short bias was not evident under saline conditions, as for the rats in the tone group, then overall accuracy was reduced by amphetamine but no choose-short effect was evident.

These findings are in agreement with those previously reported for pigeons (Stubbs \& Thomas, 1974) and rats (Rapp \& Robbins, 1976) in that there is no evidence that amphetamine simply results in an overestimation of the duration of temporal samples. To the extent that any systematic response bias is evident under amphetamine, it clearly tends to be a choose-short bias. It is difficult to account for Spetch and Treit's (1984) finding of a "chooselong" bias in pigeons under amphetamine at a 0 -sec delay. Despite the use of similar procedures, such a bias was not observed in either the present study or that of Stubbs and Thomas (1974). However, in another respect, our data are in agreement. All studies indicate that amphetamine disrupts the accuracy of temporal discriminations in both rats and pigeons even at a $0-\mathrm{sec}$ delay. Our findings parallel those reported by Bushnell and Levin (1993) and Dunnett (1985) with respect to the effects of amphetamine on spatial delayed nonmatching-to-sample in rats. These researchers found that amphetamine reduced the intercept of the retention function for spatial information without increasing its slope. Our results are congruent with this, and they support the conclusion that amphetamine does not disrupt the maintenance of either spatial or temporal sample information as much as its encoding prior to the delay.
In the study by Bushnell and Levin (1993), amphetamine reduced the accuracy of delayed nonmatching-toposition without affecting motor function or the rats' ability to respond to a cued lever (visual discrimination accuracy). The maximum dose of $d$-amphetamine used was $1.0 \mathrm{mg} / \mathrm{kg}$. Although slightly larger doses of amphetamine were used in the present study, it is worthwhile noting that the $2.0-\mathrm{mg} / \mathrm{kg}$ dose of amphetamine that disrupted the accuracy of temporal matching in pigeons had no effect on color matching. This replicates the relative insensitivity of delayed color matching to disruption by amphetamine reported by Teal and Evans (1982). Spatial and temporal delayed discriminations appear to be particularly sensitive to disruption by amphetamine at doses equal to or below $2.0 \mathrm{mg} / \mathrm{kg}$.

Previous research (Maricq \& Church, 1983; Maricq et al., 1981; Meck, 1983; Meck \& Church, 1987) has provided considerable support for the biological basis of some of the parameters of an information-processing model of time perception (Church, 1984). According to this research, drugs that affect the action of the neurotransmitter dopamine change the speed of the clock, whereas drugs that affect the action of the neurotransmitter acetylcholine change the memory storage constant (Meck, 1983). Other manipulations, such as the administration of nutrients prior to a test session (Meck \& Church, 1987) or the physical intensity of the stimulus being timed (Wilkie, 1987) can act like drugs in modifying the psychological processes involved in time perception. Church and Broadbent (1990) have suggested that the effect of biological manipulations on time perception can also be accounted for by a connectionist model in which storage and retrieval processes are separated. The clock of the information-processing model (pacemaker, switch, and accumulator) is replaced in the connectionist model by a set of oscillators, switches, and status indictors. According to Church and Broadbent, a drug that affected both storage and retrieval oscillators would produce an effect that resembles a change in clock speed. Thus, according to the connectionist model, dopamine regulates the speed of both the storage and the retrieval oscillators, whereas acetylcholine regulates the speed of only the storage oscillator. Our findings and those of Rapp and Robbins (1976) and Stubbs and Thomas (1974) demonstrate that amphetamine-induced impairment of temporal discrimination performance involves more than simply direct influences on internal clock processes. The connectionist model of time perception proposed by Church and Broadbent (1990) has just as much difficulty with our findings as does the informationprocessing model.

The present studies were designed to extend the current knowledge of the effects of amphetamine on both time perception and memory for durations in both pigeons (Experiment 1) and rats (Experiment 2). In both experiments, amphetamine reduced the accuracy of temporal discrimination even at the 0 -sec delay, but did not increase the slope of the retention functions. In addition, in neither experiment was there any evidence that amphetamine 
produced an overestimation of perceived sample duration. Amphetamine predominantly affects temporal memory in both pigeons and rats by disrupting attention to temporal samples rather than by simply increasing the speed of the internal clock.

\section{REFERENCES}

BAUER, R. H., \& Fuster, J. M. (1978). Effects of $d$-amphetamine and prefrontal cortical cooling on delayed matching-to-sample behavior. Pharmacology. Biochemistry \& Behavior, 8, 243-249.

BeAtTy, W. W., \& BierLey, R. A. (1985). Scopolamine degrades spatial working memory but spares spatial reference memory: Dissimilarity of anticholinergic effect and restriction of distal visual cues. Pharmacology, Biochemistry \& Behavior, 23, 1-6.

BeAtTy, W. W., Bierley, R. A., \& Boyd, J. (1984). Amphetamine disrupts both working and reference memories of rats trained in a radial maze. Behavioral \& Neural Biology, 42, 169-176.

BeAtTy, W. W., \& Rush, J. R. (1983). Retention deficit after $d$ amphetamine treatment: Memory defect or performance change? Behavioral \& Neural Biology, 37, 265-275.

BENINGER, R. J. (1983). The role of dopamine in locomotor activity and learning. Brain Research Review, 6, 173-196.

Burešoví, O., Bolhuis, J. J., \& BurEš, J. (1986). Differential effects of cholinergic blockade on performance of rats in the water tank navigation task and in the radial water maze. Behavioral Neuroscience, $100,476-482$.

BuREŠoví, O., \& BUREŠ, J. (1982). Radial maze as a tool for assessing the effect of drugs on the working memory of rats. Psychopharmacology, 77, 268-271.

Bushnel., P. J., \& LEvin, E. D. (1993). Effects of dopaminergic drugs on working and reference memory in rats. Pharmacology, Biochemistry \& Behavior, 45, 765-776.

Church, R. M. (1984). Properties of the internal clock. In J. Gibbon \& L. Allan (Eds.), Timing and time perception (Annals of the New York Academy of Sciences, Vol. 423, pp. 566-582). New York: New York Academy of Sciences.

Church, R. M., \& Broadbent, H. A. (1990). Alternative representations of time, number and rate. Cognition, 37, 55-81.

DUNNETT, S. B. (1985). Comparative effects of cholinergic drugs and lesions of the nucleus basalis or fimbria-fornix on delayed matching in rats. Psychopharmacology, 87, 357-363.

Eckerman, D. A., Gordon, W. A., Edwards, J. D., MacPhail, R. C., \& GAGE, M. I. (1980). Effects of scopolamine, pentobarbitol, and amphetamine on radial arm maze performance in the rat. Pharmacology, Biochemistry \& Behavior, 12, 595-602.

Fischman, M. W. (1987). Cocaine and the amphetamines. In H. Y. Meltzer (Ed.), Psychopharmacology: The third generation of progress (pp. 1543-1553). New York: Raven Press.

HAYCOCK, J. W., VAN BUSKIRK, R., \& GOLD, P. E. (1977). Effects of post-training epinephrine injections on retention of avoidance training in mice. Behavioral Biology, 20, 197-204.

JANAK, P. H., \& MARTINEZ, J. L., JR. (1992). Cocaine and amphetamine facilitate retention of jump-up responding in rats. Pharmacology, Biochemistry \& Behavior, 41, 837-840.

GLICK, S. D., \& JARVIK, M. E. (1969). Impairment by $d$-amphetamine of delayed matching performance in monkeys. Journal of Pharmacology \& Experimental Therapeutics, 169, 1-6.

GoOdman, I. J. (1981). Amphetamine and apomorphine induced stereotyped behavior in adult pigeons. Pharmacology, Biochemistry \& Behavior, 15, 701-704.

Grant, D. S., \& SPeTch, M. (1991). Pigeons' memory for event duration: Differences between choice and successive matching tasks. Learning \& Motivation, 22, 180-199.

GraNT, D. S., \& SPETCH, M. (1993). Analogical and nonanalogical coding of samples differing in duration in a choice-matching task in pigeons. Journal of Experimental Psychology: Animal Behavior Processes, 19, 15-25.
Kesner, R. P. Bierley, R. A., \& Pebbles, P. (1981). Short-term memory: The role of $d$-amphetamine. Pharmacology. Biochemistry \& Behavior, 15, 673-676.

MaricQ, A. V., \& ChURCh, R. M. (1983). The differential effects of haloperidol and methamphetamine on time estimation in the rat. Psychopharmacology, 79, 10-15.

MaricQ, A. V., RoberTs, S., \& CHURCh, R. M. (1981). Methamphetamine and time estimation. Journal of Experimental Psychology: Animal Behavior Processes, 7, 18-30.

Martinez, J. L., JR., Jensen, R. A., Messing, R. B., Vasquez, B. J., Soumireu-Mourat, B., Geddes, D., Liang, K. C., \& MCGaugh, J. L. (1980). Central and peripheral actions of amphetamine on memory storage. Brain Research, 182, 157-166.

MECK, W. H. (1983). Selective adjustment of the speed of internal clock and memory processes. Journal of Experimental Psychology: Animal Behavior Processes, 9, 171-201.

Meck, W. H., \& ChurCh, R. M. (1987). Nutrients that modify the speed of internal clock and memory storage processes. Behavioral Neuroscience, 101, 465-475.

Packard, M. G., \& White, N. M. (1989). Memory facilitation produced by dopamine agonists: Role of receptor subtype and mnemonic requirements. Pharmacology, Biochemistry \& Behavior, 33, 511-518.

RAPP, D. L., \& RoBBins, T. W. (1976). The effects of $d$-amphetamine on temporal discrimination in the rat. Psychopharmacology, 51,91-100.

REBEC, G. V., \& BASHORE, T. R. (1984). Critical issues in assessing the behavioral effects of amphetamine. Neuroscience \& Biobehavioral Reviews, 8, 153-159.

Santi, A., Bogles, J., \& Petelka, S. (1988). The effect of scopolamine and physostigmine on working and reference memory in pigeons. Behavioral \& Neural Biology, 49, 61-73.

SANTI, A., WeISE, L., \& KUIPER, D. (1995). Memory for event duration in rats. Learning \& Motivation, 26, 83-100.

SPETCH, M. L. (1987). Systematic errors in pigeons' memory for event duration: Interaction between training and test delay. Animal Learning \& Behavior, 15, 1-5.

SPETCH, M. L., \& SinhA, S. S. (1989). Proactive effects in pigeons' memory for event durations: Evidence for analogical retention. Journal of Experimental Psychology: Animal Behavior Processes, 15, 347-357.

SPETCH, M. L., \& TREIT, D. (1984). The effect of $d$-amphetamine on short-term memory for time in pigeons. Pharmacology, Biochemistry \& Behavior, 21, 663-666.

SPETCH, M. L., \& WILKIE, D. M. (1983). Subjective shortening: A model of pigeons' memory for event duration. Journal of Experimental Psychology: Animal Behavior Processes, 9, 14-30.

StrupP, B. J., Bunsey, M., LeVitsky, D., \& KeSLER, M. (1991). Timedependent effects of post-trial amphetamine treatment in rats: Evidence for enhanced storage of representational memory. Behavioral \& Neural Biology, 56, 62-76.

StuBbs, D. A., \& ThOMAS, J. R. (1974). Discrimination of stimulus duration and $d$-amphetamine in pigeons: A psychophysical analysis. Psychopharmacologia, 36, 313-322.

Teal, J. J., \& Evans, H. L. (1982). Effects of DAVP, a vasopressin ana$\log$, on delayed matching behavior in the pigeon. Pharmacology, Biochemistry \& Behavior, 17, 1123-1127.

WILKIE, D. M. (1987). Stimulus intensity affects pigeons' timing behavior: Implications for an internal clock model. Animal Learning \& Behavior, 15, 35-39.

WILKIE, D. M., \& WILLSON, R. J. (1990). Discriminal distance analysis supports the hypothesis that pigeons retrospectively encode event duration. Animal Learning \& Behavior, 18, 124-132.

Wirsching, B. A., Beninger, R. J., Jhamandas, K., Boegman, R. J., \& EL-DEFRAWY, S. R. (1984). Differential effects of scopolamine on working and reference memory of rats in the radial maze. Pharmacology, Biochemistry \& Behavior, 20, 659-662.

(Manuscript received July 25, 1994; revision accepted for publication January 20, 1995.) 